\title{
Development of a stock trading system based on a neural network using highly volatile stock price patterns
}

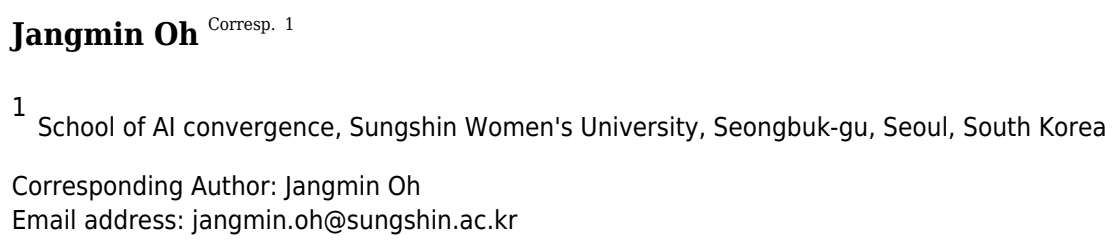

This paper proposes a pattern-based stock trading system using ANN-based deep learning and utilizing the results to analyze and forecast highly volatile stock price patterns. Three highly volatile price patterns containing at least a record of the price hitting the daily ceiling in the recent trading days are defined. The implications of each pattern are briefly analyzed using chart examples. The training of the neural network was conducted with stock data filtered in three patterns and trading signals were generated using the prediction results of those neural networks. Using data from the KOSPI and KOSDAQ markets, It was found that that the proposed pattern-based trading system can achieve better trading performances than domestic and overseas stock indices. The significance of this study is the development of a stock price prediction model that exceeds the market index to help overcome the continued freezing of interest rates in Korea. Also, the results of this study can help investors who fail to invest in stocks due to the information gap. 
1 Development of a stock trading system based on a

2 neural network using highly volatile stock price

3 patterns

4

5

6

7

8

9

10

11

12

13

14

15

16

17

18

19

20

21

22

23

24

25

26

27

28

29

30

31

32

33

34

35

36

37

38

39

Jangmin $\mathrm{Oh}^{1}$

${ }^{1}$ School of AI Convergence, Sungshin Women's University, 10 Dongsomun-ro 20da-gil

Seongbuk-gu Seoul, 136-051, Korea

Corresponding Author:

Jangmin $\mathrm{Oh}^{1}$

10 Dongsomun-ro 20da-gil Seongbuk-gu Seoul, 136-051, Korea

Email address: jangmin.oh@sungshin.ac.kr

\section{Abstract}

This paper proposes a pattern-based stock trading system using ANN-based deep learning and utilizing the results to analyze and forecast highly volatile stock price patterns. Three highly volatile price patterns containing at least a record of the price hitting the daily ceiling in the recent trading days are defined. The implications of each pattern are briefly analyzed using chart examples. The training of the neural network was conducted with stock data filtered in three patterns and trading signals were generated using the prediction results of those neural networks. Using data from the KOSPI and KOSDAQ markets, It was found that that the proposed patternbased trading system can achieve better trading performances than domestic and overseas stock indices. The significance of this study is the development of a stock price prediction model that exceeds the market index to help overcome the continued freezing of interest rates in Korea. Also, the results of this study can help investors who fail to invest in stocks due to the information gap.

\section{Introduction}

Predicting stock prices has long been of interest to many related fields including economics, mathematics, physics, and computer science. There is an ongoing debate about whether or not it is possible to predict stock prices and, if it is possible, how much these predictions can outperform the market. However, the field of AI (artificial intelligence) has recently reported price forecasting techniques with the application of various machine learning techniques that show a significant level of statistical confidence (Hsu, 2011; Hadavandi et al., 2010; G.Armano et al., 2005; Ding et al., 2015; Jiang, 2021). A number of studies building intelligent trading systems have also been conducted based on the results of these AI price forecasting techniques (Lin et al., 2011; O et al., 2004; Song et al., 2020). 
40 O et al. $(2004,2006)$ concluded that trading performance can be additionally improved by

41 training and utilizing independent predictors for different stock price patterns.

42 Most of the existing stock price prediction technical analyses have based the input features on

43 the moving average (MA) stock price, which can effectively express the recent trends of price

44 fluctuations. For example, the MACD (moving average convergence divergence) utilizes the

45 difference between the long term and short-term moving average to represent the convergence

46 and divergence of the moving average values.

47 O et al. (2006) performed pattern-defined predictions using patterns related to a crossover,

48 reversal into an uptrend, and reversal into a downtrend among 5-day, 10-day and 20-day moving

49 average lines.

50 However, all of the MA-based technical indicators, including the MACD, have a 'time-lag'

51 limitation because buy and sell signals are mostly generated after price trends have already been

52 developed. This study will attempt to predict highly volatile stock price patterns by introducing

53 the concept of the 'upper limit price,' which is defined independently from the moving average.

54 This study will also utilize Japanese candlestick indicators and more short-term technical

55 indicators to complement the time-lag problem.

56 In the Japanese candlestick indicator, a candlestick summarizes the intraday variations of a stock

57 price, expressing the differences between the opening price, highest, lowest, and closing prices,

58 through which the most recent price fluctuations can be summarized more closely.

59 According to various empirical analyses of the Korean stock market, the Korean stock market

60 shows market inefficiency due to information asymmetry (Lee, 2007; Bark, 1991). Although

61 market inefficiency is lower than that of larger foreign stock markets, there is still an issue of the

62 information gap. Therefore, this paper suggest that special investment and analysis information

63 to overcome market inefficiency. However, since technical analysis indicators are price- and

64 chart-based information that many people already know, a new chart analysis technique is

65 needed.

66 The efficient market hypothesis asserted by Fama (1965) is rejected if it exceeds the market rate

67 of return using specific information. This study proposes a new 'highly volatile stock price

68 pattern' that does not yet exist in technical analysis. Using the pattern proposed in this paper, it is

69 possible to develop a predictive model that exceeds the market return. The results of this study

70 are in conflict with the efficient market hypothesis.

71 In summary, this study assumes market inefficiency in the Korean stock market and provides

72 new information that is expected to affect price fluctuations. The highly volatile stock price

73 pattern is defined by the relationship between the 'upper limit' and stock price in the Korean

74 stock market. Through fund simulation it was found that investors can obtain efficient returns

75 through a deep learning stock price prediction model using highly volatile patterns.

76 It was also found that it was difficult to predict stock prices by only analyzing simple charts such

77 as moving averages, so a definition of a particular pattern of variation is needed.. This pattern

78 can be found when there are stock prices that show an upper limit. This pattern can also appear

79 over various periods of time even when the chart shows the characteristics of a random walk. 
80 The experiment was conducted based on related research that the Korean stock market shows

81

82

83

84

85

86

87

88

89

90

91

92

93

94

95

96

97

98

99

100

101

102

103

104

105

106

107

108

109

110

111

112

113

114

115

116

117

118

119

more trend changes than random work characteristics (Aggarwal, 2018; Ryoo \& Smith, 2002; Ayadi \& Pyun, 1994).

Studies related to stock market analysis from China, India, and Mongolia, which are similar to Korea, were also unable to prove the random walk theory according to the stock market (Han et al., 2019; Damdindori et al. 2016).

The present study will show that the proposed stock trading system can achieve better trading performances than domestic and overseas stock indices by performing pattern-specific training on highly volatile stock price patterns. The experiments were conducted on the stock variation data extracted from the price data of approximately 2,000 stocks listed in the KOSPI and KOSDAQ markets. The limitations of existing studies and the strengths of this study are as follows.

\section{Limitations of existing studies}

1. Limitations of Profitability Verification: Existing studies suggest only accuracy or prediction error using stock price prediction models. However, the actual performance of the stock price prediction model requires return verification.

2. Limitations of using simple input features: Existing studies have used very simple input features such as simple price features, volume, price rate of change and so on. Simple input features are limited and using more advanced input features can greatly influence the performance of predictive models.

3. Limitations on prediction accuracy: Most of the predictive models in existing studies have not achieved high accuracy.

\section{Strengths of this study}

This study conducted a clear performance verification through yield comparison with several indices. In addition, although a simple deep neural network was used, advanced input features improved the performance of the stock price prediction model. Although there are time series models such as LSTM, this study uses high-volatile pattern filtering input features. As a result, stock price data of a portion without the corresponding pattern is partially filtered. Therefore, the continuity of time series data is insignificant. As a result, deep learning helped us identify the importance of input features such as high volatility patterns in predicting stock price. The deep neural network we used is a rather simple structure, but its sufficient performance has been verified. The novelty of this study is summarized as follows.

- First, an advanced filtering technology that can improve the performance of the stock price prediction model was proposed. The pattern of stock price fluctuations proposed in this paper was created based on the results of analyzing domestic stock charts. Since it is

Peer] Comput. Sci. reviewing PDF | (CS-2021:11:67714:1:1:NEW 27 Jan 2022) 
a pattern generated based on the actual chart, it reflects the market well and shows excellent performance compared to the existing moving average-based pattern.

- Second, it enables investors to make comfortable investments without daily data analysis. By using the neural network model, a lot of data can be handled at once, and the prediction results are reliable results that have been verified through a sufficient period of fund simulation.

- Third, advanced filtering technology enables sufficient stock price prediction even in a simple deep learning model. Most of the existing studies focus on the structure of the model when conducting deep learning stock price prediction studies. For example, performance is compared using multiple models for the same data. However, in addition to the issue of selecting the structure of the model, the composition of data and the importance of filtering algorithms are proposed through this study.

This paper is organized as follows: "Related Works" describes related studies; "Background" describes the knowledge used prior to the main methodology of this study; in "Materials \& Methods," the moving average-based patterns typically utilized in existing studies will be introduced, the formal definition of the three highly volatile stock price patterns will be presented, and the meanings of the individual patterns will be described through chart demonstrations; in "Experiments," the input features and the target value of the neural network learning system will be described; in "Results of experiments," the experimental results will be presented; and, finally, future research directions will be suggested in the "Conclusion."

\section{Related Works}

Researches related to stock price prediction have traditionally been conducted using ARIMA (Benvenuto D et al, 2019; Ariyo et al, 2014), Regression (Refenes A et al, 1994; Yang H et al, 2002), and Bayesian (Pella J \& Masuda M, 2001) to reflect the characteristics of time series data. ARIMA is a statistical model widely used in the financial sector. However, it is a model that is used exclusively for short-term predictions, and has the disadvantage that it is difficult to confirm long-term investment performance. Since stock price prediction is closely related to profits, direct investment in a short-term verified model can lead to risks. In addition, for the reason that the amount of stock data accumulated from the past is very vast, a model that can handle large amounts of data is needed. In this regard, there is also a study result that predicts the index using the ARIMA model has an accuracy of up to 38\% (Devi et al, 2013). However, the prediction accuracy was very low to carry out actual investments using this model. Baysian is a model that can perform classification based on probabilistic theory and used to predict stock prices in the past. However, with the recent development of artificial neural networks, it is widely used as a comparative model. Baysian is also evaluated as not suitable for mid or long-term prediction, such as the ARIMA model. In related studies using the Bayesian model, predictions were performed with up to $78 \%$ accuracy (Malagrino et al, 2018). This was 
160 better performance than the ARIMA model, but it showed lower values than the neural network 161 model to be described below.

162 Above all, these existing predictive models often misinterpret information due to underfitting 163 and overfitting problems, so they often do not help much in decision-making activities for stock 164 price prediction. In addition, it has already been proven that neural networks perform better than 165 traditional methods (Bustos \& Pomares-Quimbaya, 2020). For the above reasons, neural 166 network-based technique has been used a lot in recent stock price prediction.

167 Representatively, there is a study using Elliott Wave Indicator as a stock price prediction study 168 using neural networks (Lakshminarayanan et al, 2006). This study used data based on technical 169 analysis, and the model accuracy was $93.83 \%$. This study used its own technical indicators and made predictions for five stocks. Based on these findings, it is possible to assume that more advanced input features can lead to improved model performance. Several other papers similar to this paper used machine learning techniques such as regression and SVM, models such as CNN and LSTM, or ensemble techniques to predict stock prices

174 (Oncharoen \& Vateekul, 2018; Liu \& Wang, 2018; Jiang, 2021).

175 Cao \& Wang (2019) attempted to predict the stock index of various countries using the CNN application model. Only historical data was used as input features, and the down-sampling and convolution techniques belonging to $\mathrm{CNN}$ were mainly used to improve stock price prediction performance. As a result, they suggested that the CNN-SVM mixed model had the best performance. However, simple input features were used, and the exact performance and return of the model were not verified, so it was difficult to confirm the actual performance of the CNNSVM model.

Another study (Guang et al., 2019) looked at the rate of the return of the stock price forecast

183

184

185

186 model. This model is not a period-specific return, but an absolute return that does not take into account investments and assets that may vary from person to person. In addition, the profits earned have no comparison with the stock index, which can be regarded as a rate of return due to market rise.

A similar study (Pang et al., 2020) included word-embedding techniques such as LSTM (Long Short-Term Memory). Here stock data, which is a time series characteristic, is referred to as a stock vector, and is used as an input feature. The model they developed tried to predict the Shanghai Stock Index and showed an accuracy of about $57 \%$, but the study did not have a comparison of returns through prediction. High volatile stock price prediction model derived higher accuracy than the model presented by Pang et al. In addition, only a few studies (Feng et al., 2018; Araujo et al., 2019) using various in-depth models have both derived returns and evaluated their performance, and most studies cannot guarantee a clear return because they provide no comparison with the stock index.

Recent evidence suggests that input feature selection is very important in model learning. The selection of key input features can lead to improved (Hooshmand \& Gad, 2020). Accordingly, in this paper, the study was conducted with a focus on data composition and novelty rather than the structure of the model. 


\section{Background}

202

203

204

205

206

207

208

209

210

211

212

213

214

215

216

217

218

219

220

221

222

223

224

225

226

227

228

229

230

231

232

233

234

235

236

\section{MA (Moving Average)-based patterns}

Before examining the high volatile stock price pattern using the Japanese candlestick indicator, which is the focus in this study, the meaning and limitations of the four MA-based patterns presented in related studies are examined, taking the 'divergence' pattern and 'reversal' pattern of MA (Moving Average) as examples. (O et al., 2006) that defined and used the patterns based on the moving average. The moving average is the average stock price over a period of time and is used to summarize stock price trends. Moving average is also denoted by $M A$ in which 5, 10, 20 days and so on are used as the window of time; as an example, the 5-day moving average of stock $s$ at trading day $t$ can be calculated as follows:

$$
M A 5_{t}^{s}=\frac{1}{5} \sum_{k=0}^{4} \text { close }_{t-k}^{s}
$$

In the same way as above, the volume can also be calculated as the moving average of the volume, and the 5-day volume moving average of stock trading day can be counted as follows:

$$
V M A 5_{t}^{s}=\frac{1}{5} \sum_{k=0}^{4} \text { volume }_{t-k}^{s}
$$

where $c l o s e_{t}^{s}$ is the closing price of the trading day $t$. The slope of the line connecting a moving average to another moving average is denoted as Grad and can be calculated using the following Equation (3):

$$
\operatorname{Grad}_{t}^{s}=\frac{M A 5_{t}^{s}-M A 5_{t-1}^{s}}{M A 5_{t}^{s}}
$$

Equation (4) defines the training target set $D_{\text {bear }}$ that corresponds to the divergence pattern. Divergence refers to when the short-term moving average is located relatively below the longerterm moving averages, resulting from the continuation of the downward trend of the stock price for a considerable period of time. Equation (4) represents that the 5-day moving average is smaller than the 10-day moving average and the 10-day moving average is smaller than the 20day moving average:

$$
D_{\text {bear }}=\left\{\left(x_{s, t}, o_{s, t}\right) \mid M A 5_{t}^{s}<M A 10_{t}^{s}<M A 20_{t}^{s}, s \in \alpha, t \in \beta\right\}
$$

where $x_{s, t}$ is a vector of the input feature, and $o_{s, t}$ is the target value representing the price fluctuation after the occurrence of the pattern; $\alpha$ and $\beta$ are the entire stock set and the entire trading day set, respectively. Figure 1 is an example of the charts corresponding to a divergence pattern. The description of the graph in Figure 1 is as follows. Sixteen trading days from A to B correspond to the divergence pattern. In this case, relatively steep price rises were shown around point B; but, in general, even if a rebound was to appear, the rise would not be that large. Equation (5) defines the training target set $D_{T U}$ that corresponds to the reversal to uptrend pattern. Reversal to uptrend means that one of the moving average lines reverses from a 
237 downtrend to an uptrend; trading day A, B, C in Figure 2 show reversal to uptrend patterns.

238 Among these, A shows the case where the 5-day $M A$ line reversed to an uptrend due to the sharp 239 rise of the stock price.

240 This case shows the weakness of MA as a prediction indicator because the price had already 241 risen before the trading signal was issued; the phenomenon of generating the signals after the 242 price movement has already occurred is called 'time-lag'. This MA-based pattern arises very 243 frequently so it has the strength of using a large amount of training data, but time lag decreases 244 this pattern's predictive ability.

245

246

247

248

249

250

251

252

253

254

255

256

257

258

259

260

261

262

263

264

265

266

267

268

269

270

271

$$
\begin{aligned}
& D_{T U}=\left\{\left(x_{s, t}, o_{s, t}\right) \mid\left(\operatorname{Grad}_{t-1}^{s}<0 \& \& \operatorname{Grad}_{t}^{s}>0\right)\right. \\
& \|\left(\operatorname{Grad} 10_{t-1}^{s}<0 \& \& \operatorname{Grad} 10_{t}^{s}>0\right), \\
& \left.\|\left(\operatorname{Grad} 20_{t-1}^{s}<0 \& \& \operatorname{Grad} 20_{t}^{s}>0\right), s \in \alpha, t \in \beta\right\}
\end{aligned}
$$

\section{Materials \& Methods}

\section{Highly volatile stock price patterns}

This section describes a technique for filtering data showing a high volatile stock price pattern on a stock chart. There are a total of three filtering algorithms, and using them, data with high fluctuation patterns form a cluster. Machine learning-based algorithms such as k-means were not necessarily used when creating clusters. This is because the time it takes to create a cluster is very short compared to similar studies (Alguliyev et al, 2019).

In the pattern-based stock trading system, multiple independent predictors are trained on the data clustered in line with the stock price patterns and employed in the final trading. In this paper, the highly volatile stock price patterns will be defined and employed as a way to achieve more predictability as an extension of pattern-based prediction techniques. Korean stock markets set the legal limits of price fluctuations in a day; both the KOSPI and KOSDAQ markets apply \pm $15 \%$ of the previous day's closing price to the price fluctuation limits. In general, the 'upper limit' refers to when the closing price of a particular trading day is closest to a $15 \%$ rise from the previous day's closing price, or about a $14 \%$ rise in the price depending on the price band of the item. In Equation (6), Sanghan ${ }_{t}^{s}$ represents the closing price at the upper limit of stock $s$ on trading day $t$ and is defined as follows:

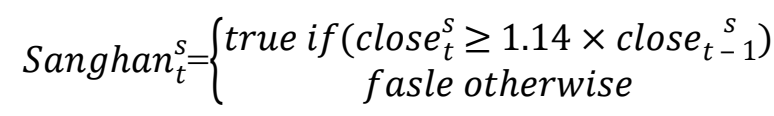

where $\operatorname{clos} e_{t}^{S}$ refers to the closing price. The three highly volatile stock price patterns are defined based on the definition of the upper limit. If the price of a particular stock has risen to the upper 
272 limit, the price volatility of the subsequent trading days is bound to be expanded. The rise of the 273 price to the upper limit should be accompanied by large transaction volume because a short-term 274 surge and plunge may occur due to the collective psychology of the trading public. The highly 275 volatile stock price patterns in this paper deal only with the case where price adjustments were 276 made 1 to 2 trading days after the upper limit price appeared. The time target used for predictions 277 was when both the first rising wave, represented by the rising to the upper limit price, and the 278 first falling wave, represented by the adjustment, have been completed so extreme volatilities 279 have been relaxed. High volatile stock price patterns were found by chart analysis experts who 280 analyzed charts over the years. This pattern is actually mainly seen in mid- to low-priced stocks 281 in the Korean stock market. Investors have to look directly at a vast amount of data to utilize this 282 pattern for real investment. However, if the prediction model using deep learning is properly 283 defined, it is easy to predict when the price rises after a certain pattern, and even check whether it 284 can actually make profits.

285

286

287

288

289

290

291

292

293

294

295

296

297

298

299

300

301

302

303

304

302

\section{Adjustment pattern with one candlestick after consecutive upper limits ( $p 1)$}

An adjustment pattern with 1 candlestick after consecutive upper limits is when the first rising wave is so strong that the upper limit prices appear consecutively; it can be calculated using the equation below (7). open ${ }_{t}^{s}$ represents the open price of stock s on trading day t. Figure 3 shows an example of $p 1$ where (a) is a normal example in which a $p 1$ pattern occurs and the upper limit price appears next day, and (b) is a counter example where the empty rectangular, called a negative candlestick, represents when the closing price is lower than the opening price, meaning that the price became lower after intraday trading. The positive candlestick filled with the grey color is the opposite. The last condition of the condition statement means that the candlestick in the pattern formation for the day should be a negative candlestick or should be a positive candlestick of less than $5 \%$ of the difference between the opening price and the closing price. If the positive candlestick is larger by a difference of more than $5 \%$, the price is considered adjusted.

$$
p 1_{t}^{s}=\left\{\begin{array}{c}
\text { true if }\left(\text { Sanghan }_{t-2}^{s}=\right.\text { true and Sanghan } \\
t-1 \\
\text { and } \left.\frac{\left(\text { closese }_{t}^{s}-\text { open }_{t}^{s}\right)}{\text { close } e_{t}^{s}}<0.05\right) \\
\text { false oterwise }
\end{array}\right.
$$


305

306

307

308

309

310

311

\section{2}

313

314

315

316

317

318

319

320

An adjustment pattern with one candlestick after a single upper limit is when the upper limit price condition is replaced by the single upper limit price; Figure 4 shows a normal example and a counter example of the case. Due to the relatively weaker rising intensity, it is estimated that the ratio of normal examples is likely to be rather lower than $\mathrm{p} 1$ patterns.

$$
p 2_{t}^{s}=\left\{\begin{array}{c}
\text { true if }\left(\text { Sanghan }_{t-2}^{s}=\right.\text { false and Sanghan } \\
{ }_{t-1}^{s}=\text { true } \\
\text { and } \left.\frac{\left(\text { close }_{t}^{s}-\text { open }_{t}^{s}\right)}{\text { close } e_{t}^{s}}<0.05\right) \\
\text { false oterwise }
\end{array}\right.
$$

\section{Adjustment pattern with two candlesticks after upper limits (p3)}

An adjustment pattern with two candlesticks after the upper limits is the case in which the price has been adjusted over two trading days after the upper limit price, or when a negative candlestick or a small positive candlestick (less than $5 \%$ in size) forms after $p 1$ or $p 2$ is formed. Figure 5 shows the examples of $p 3$. The last candlestick on each chart of (a) and (b) is the price fluctuation immediately after the pattern occurs.

$$
p 3_{t}^{s}=\left\{\begin{array}{c}
\text { true if }\left(\text { Sanghan }_{t-1}{ }^{s}=\text { true and } \frac{\left(\text { close }_{t-1}^{s}-\text { open }_{t-1}^{s}\right)}{\operatorname{close}_{t-1}^{s}}<0.05\right. \\
\text { and } \frac{\left(\text { close }_{t}^{s}-\text { open }_{t}^{s}\right)}{\text { close }_{t}^{s}}<0.05 \\
\text { false oterwise }
\end{array}\right.
$$

These examples of the three patterns examined above imply that price fluctuations after the pattern occurs can vary depending on the slope of the moving average and the form of the candlestick. As an example, the under tail is attached to the last candlestick in all the patterns shown in the three normal examples. This means that the stock ended with the emergence of buying powers leading the rebound in price after trading hours and is more likely to be bullish the next day. Since the various factors act in combination on the direction of the stock price after the appearance of the pattern, the neural network training that will be described in the next section is needed to utilize these patterns in the trading system.

\section{Experiments}

\section{Input features configuration for neural network}

In order to train the neural networks for future price predictions for each pattern presented in the previous sections, the input feature set constructing an input vector $\boldsymbol{x}_{\boldsymbol{s}, \boldsymbol{t}}$ was used for the input to the neural network from the training data and the target value corresponding to the desired output was defined. Disparity, representing the distance between $\boldsymbol{M A}$ and the current price, is denoted as Disp and the Disp from the 5-day MA line can be calculated using the following equation: 


$$
\operatorname{Disp} 5_{t}^{S}=\frac{\operatorname{close}_{t}^{S}-M A 5_{t-1}^{s}}{M A 5_{t}^{s}}
$$

337

338

339

340

341

342

343

344

Apart from the moving average line, the input features relating to Japanese candlesticks include: $\boldsymbol{R C}$ (rate of change) in the trading day price compared to the previous day, Body, US (upper shadow), and $\boldsymbol{L S}$ (lower shadow). These are defined in equations (11) through (14), respectively:

$$
\begin{aligned}
& R C_{t}^{S}=100 \mathrm{x} \frac{\operatorname{cose}_{t}^{s}-\operatorname{close}_{t-1}^{s}}{\operatorname{clos}_{t}^{s}} \\
& \operatorname{Body}_{t}^{S}=100 \mathrm{x} \frac{\operatorname{open}_{t}^{s}-\operatorname{close}_{t-1}^{s}}{\min \left(\text { open }_{t}^{s}, \operatorname{close}_{t}^{s}\right)} \\
& U S_{t}^{S}=100 \mathrm{x} \frac{\operatorname{high}_{t}^{s}-\max \left(o p e n_{t}^{s} \operatorname{close}_{t}^{S}\right)}{\max \left(\operatorname{open}_{t}^{s} \text { close }_{t}^{s}\right)} \\
& L S_{t}^{S}=100 \mathrm{x} \frac{\min \left(\text { open }_{t}^{s}, \operatorname{cose}_{t}^{s}\right)-\operatorname{low}_{t}^{s}}{\min \left(\text { open }_{t}^{s}, \operatorname{clos}_{t}^{s}\right)}
\end{aligned}
$$

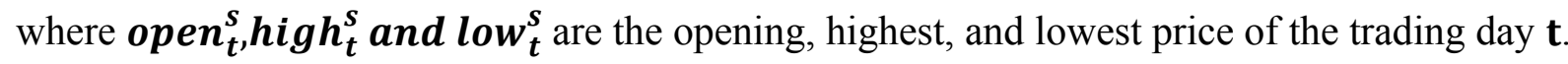
The input vector $\boldsymbol{x}_{\boldsymbol{s}, \boldsymbol{t}}$ of each predictor, including these indicators, is as follows:

$$
\begin{aligned}
& x_{s, t}=\left(R C_{t}^{s}, R_{t-1}^{s}, R C_{t-2}^{s}, \operatorname{Body}_{t}^{s}, \operatorname{Body}_{t-1}^{s}, \operatorname{Body}_{t-2}^{s}, U S_{t}^{s}, L S_{t}^{s}, \operatorname{Grad5}_{t}^{s}\right. \text {, } \\
& \operatorname{Grad10}_{t}^{s}, \operatorname{Grad20}_{t}^{s}, \operatorname{Disp} 5_{t}^{s}, \operatorname{Disp} 10_{t}^{s}, \operatorname{Disp20} t_{t}^{s}, \operatorname{VGrad}_{t}^{s}, \operatorname{VGrad10}_{t}^{s} \text {, } \\
& \operatorname{VGrad20}{ }_{t}^{s}, \operatorname{VDisp} 5_{t}^{s}, \operatorname{VDisp} 10_{t}^{s}, \operatorname{VDisp} 20_{t}^{S} \text { ) }
\end{aligned}
$$

where VGrad is the slope of the volume moving average line. VDisp is the difference between the volume moving average and the total volume; these two indicators can be calculated by entering the total volume instead of the close price in the equations of the Grad and Disp. Each input feature should be normalized as a value between 0 and 1 before used.

\section{Experimental process and environment}

The first of the stock price prediction process takes data from the stock database for a certain period of time. Thereafter, the data of the part showing the high volatile pattern is filtered and the input feature is calculated. The calculated result is saved as a text file and is used for deep learning. The text file consists of training, validation, testing, and fund simulation files, and finally the model outputs dates and stocks that are expected to rise by more than $10 \%$. The stock database stores daily KOSPI/KOSDAQ data from October 1990 and it updates data every day. Therefore, even if the composition of the stock index changes, the changed information is newly added to the database, so it is possible to predict the changed index. A prediction model using the neural network structures as shown in Figure 6 below was trained using 20 input features and binary classified target vectors. The target vector uses a binary 
366 classification format, and if the price rises by more than $10 \%$ within 5 days, it will be marked

$367[0,1]$ otherwise $[1,0]$.

368 Experiments will be conducted on a desktop with 18.04 versions of Ubuntu with RTX 3070 9GB

369 graphics card. The model was constructed using Keras in Tensorflow 2.0 (Abadi et al., 2016) and

370 the hidden layer was composed of three hidden layers. In addition, the use of detailed parameters

371 is as follows. Tanh was used as an activation function for each layer, and the learning rate was

372 set to 0.01 . In addition, the dropout ratio was set to 0.5 .

373

374 Experiment Results

375

376

377

378

379

380

381

382

383

384

385

386

387

388

389

390

391

392

393

394

395

396

397

398

399

400

401

402

\section{Performance evaluation of prediction model}

This section will present the experiment results of applying the proposed trading system to the prices of 2,268 stocks listed on the KOSDAQ and KOSPI markets of the Korean Stock Exchange. The entire dataset used in the experiment is divided into 4 sub-sets; the details are shown in Table 1. In other related studies, there are no results of measuring returns through the prediction model. In studies related to stock price prediction, not only the accuracy of the prediction model but also the measurement of the rate of profit should be conducted at the same time. In this paper, a fund simulation dataset is additionally configured for this purpose.

Training, verification, and test data are used exclusively for determining prediction models. Only the data that was not used to generate the prediction model was used for fund simulation. This process is for cross-validation and accurate rate of profit measurement.

This study was aimed only at predicting the domestic stock market, so only data from the KOSPI and KOSDAQ were used. . The total number of KOSPI and KOSDAQ stocks in Korea is about 2,436. In this study, data were collected from October 2017 to December 2020 based on KOSPI and KOSDAQ stocks, the total number of data used for training, verification, and testing, and additional fund simulations exceeded 2 million. Because of the large amount of data, other analytical techniques and theories were needed to add data from overseas stock markets. The experiments showed that as training progressed, loss decreased and accuracy increased, as shown in Figure 7. The loss calculation used MSE, and the equation is as follows. Where $\boldsymbol{N}$ is the number of samples, $\boldsymbol{P}$ is the predicted value and $\boldsymbol{A}$ is real value. Mean square error is defined as the variance between predicted and actual values (Namasudra et al, 2021).

$$
M S E=\frac{1}{N} \Sigma_{1}^{N}\left(P_{i}-A_{i}\right)^{2}
$$

Test datasets showed a slight increase in loss and a slight decrease in accuracy with each epoch. However, we can see significantly better numerical results than the training process.

The evaluation of the prediction model is shown in Table 2. The model's evaluation was performed on two criteria: accuracy and F1 score. Accuracy is a metric for the classification model as a percentage of the total predictions performed. The F1 score is the harmonic mean of 
403 precision and recall (Chakraborty et al, 2020). The equation for accuracy and fl score is as

404 follows.

405

406

407

408

409

410

411

412

413

414

415

416

417

418

419

420

421

422

423

424

425

426

427

428

429

430

431

432

433

434

435

436

437

438

439

$$
\begin{gathered}
\text { Accuracy }=100 \mathrm{x}_{\text {True Positives }+ \text { True Negatives }+ \text { False Positive }+ \text { False Negatives }}^{\text {True Positives } \text { True Negative }} \\
\text { F1 score }=2 * \frac{\text { Precision } * \text { Recall }}{\text { Precision }+ \text { Recall }}
\end{gathered}
$$

where:

Precision $=\frac{\text { True Positives }}{\text { True Positives }+ \text { False Positive }}$, Recall $=\frac{\text { True Positives }}{\text { True Positives }+ \text { False Negatives }}$

The experiments showed that the stock price prediction model using highly volatile stock price patterns finally showed an accuracy of $96.23 \%$ and an F1 score of 0.9638 . This result is slightly lower or better compared to other classification models (Agrawal et al, 2021; Ndichu et al, 2020) not stock price prediction models. However, in the case of stock price forecasts, this figure can be said to be a good result due to high uncertain volatility.

The predictors by pattern were constructed using the training data by pattern, and the optimum trading policies were selected by performing the integrated multiple simulation presented in Lee (2007), applying the 'trading policy selection set' to each predictor. Here, the integrated multiple simulations refer to the technique to find the optimal trading policies best suited to a given predictive neural network. For example, when a prediction is performed on the fund simulation set, stocks and dates that will rise more than $10 \%$ are derived. The stocks to rise consist of those with a neural network threshold of more than 0.5 . It was found that the optimum trading policy had a $20 \%$ in profit realization rate, $-12 \%$ in stop loss rate, and a holding period of 19 days.

The results of this experiment were compared with similar studies using other filtering algorithms to derive the results shown in Table 3. Highly volatile filtering algorithm defines the pattern of fluctuations in stock prices using the concept of upper limits. In comparison, the remaining three algorithms are 'Resisted plunge filtering', 'Nosedive filtering', and 'Rise stock filtering', respectively. 'Resisted plunge' refers to the type in which an ascending stock drops for a short period. 'Nosedive' literally means a slump. In this case, it is to collect stocks that have shown a period of collapse. 'Rise stock' filtering represented the long-term upward trend (Song, Y et al, 2018).

Experiments were conducted using the same data and model structure. The experimental results showed that Resisted plunge filtering achieved 72.39\% accuracy, Nosedive filtering achieved $75.11 \%$ accuracy, and Rise filtering achieved 64.93\% accuracy. In contrast, it can be seen that the highly volatile pattern filtering algorithm is $96.23 \%$, which is higher than the accuracy of the other filtering algorithm.

\section{Results of Fund simulation}


440 As a result of the fund simulation using optimal trading policies, it was able to earn better profits 441 than the domestic stock index during the same period. Industrial indicators were not predicted 442 separately because they were both included in the KOSPI and KOSDAQ already as this data 443 contains all stock data by industry. As a consequence, the number of stock trades was small 444 because the stock price pattern showing a highly volatile pattern did not appear much in the 445 fluctuation pattern of all stocks. However, when a highly volatile pattern occurs, It was found 446 that there is a high probability that a subsequent upward pattern will appear. In conclusion, 447 highly volatile stock price patterns play a big role in predicting stock price rise.

448 Additionally, to supplement the stock price prediction performance of the high volatile model 449 proposed in this paper, fund simulation results were compared it with the Nikkei 225, NYSE, and 450 NASDAQ, which are representative stock indices in Japan and the United States. The results are 451 shown in a graph with the domestic stock index in Figure 8. Even when other indices were 452 lowered, high volatile stock price prediction model showed a steady upward graph. Finally, this 453 model can earn the highest cumulative return. Figure 8 shows the percentage of returns from 454 each asset.

455 The reasons that the trading system proposed in the paper achieved better trading performance 456 than the domestic and overseas stock indices are: first, including the microscopic price change 457 processes of the most recent three days in the training input features helped train the neural 458 network training; second, defining the scope of each pattern by using more strengthened 459 constraints than the moving average pattern seems to have contributed to the improvement of 460 learning performance as well as the ultimate trading performance.

461

\section{Conclusions}

463

464

465

466

467

468

469

470

471

472

473

474

475

476

477

478

479
This paper constructed a pattern-based stock trading system which learned data corresponding to the three highly volatile stock price patterns and utilized that data for trading. The highly volatile stock price pattern can be observed over a long period of time and almost guarantees a short-term rise after the pattern occurs.

The significance of this study is the development of a stock price prediction model that exceeds market indices to overcome the continued freezing of interest rates in Korea, Japan, and the U.S. Also, the results of this study can help investors who fail to invest in stocks due to the information gap. If special analysis techniques and indicators such as high volatility patterns are proven to be effective through this research method, individual investors can use these methods in the future. In addition, a number of other patterns of variation can be added to expand the model, and if a positive return is proven, anyone can use the fund simulation for their own investments.

Additional studies will have to be conducted to achieve much better trading performances through microscopic analysis and classification of other highly volatile stock price patterns not used in this paper. Improving the input feature set used in this paper, and reflecting the variations of the periods in the target values may also help achieve better results. In addition, performance 
480

481

482

483

484

485

486

487

488

489

490

491

492

493

494

495

496

497

498

499

500

501

502

503

504

505

506

507

508

509

510

511

512

513

514

515

516

517

changes will be measured by applying the NAR Neural Network Time Series (NAR-NNTS), a recently studied model (Namasudra et al, 2021) that is suitable for data with uncertainty in future studies.

Finally, this study was conducted only using Korean stock data as the Korean stock market is very different from overseas stock markets such as those in the United States. However, in order to highlight the strengths of prediction model by performing cross-comparison with overseas stock indices, comparisons with three indices were added. Price restrictions such as upper and lower limits are usually used in Asia including China, Japan, and Thailand. This paper identified patterns inspired by Japanese candle charts and compared them with the Japanese stock index. In addition, it completed the yield comparison by adding comparison with overseas stock markets such as the U.S. As a result, the proposed model showed a higher return than the overseas market growth rate during the same period.

\section{References}

Abadi, M, Agarwal, A, Barham, P, Brevdo, E, Chen, Z, Citro, C, ... \& Zheng, X. 2016.

Tensorflow: Large-scale machine learning on heterogeneous distributed systems. arXiv preprint arXiv:1603.04467.

Aggarwal, D. 2018. Random walk model and asymmetric effect in Korean composite stock price index. Afro-Asian Journal of Finance and Accounting, 8(1), 85-104.

Agrawal, D., Minocha, S., Namasudra, S., \& Kumar, S. 2021. May. Ensemble algorithm using transfer learning for sheep breed classification. In 2021 IEEE 15th International Symposium on Applied Computational Intelligence and Informatics (SACI),199-204

Ayadi, O. F., \& Pyun, C. S. 1994. An application of variance ratio test to the Korean securities market. Journal of banking \& finance, 18(4), 643-658.

Alguliyev, R. M., Aliguliyev, R. M., \& Sukhostat, L. V. 2019. Efficient algorithm for big data clustering on single machine. CAAI Transactions on Intelligence Technology, 5(1), 9-14.

Araújo, R. D. A., Nedjah, N., Oliveira, A. L., \& Silvio, R. D. L. 2019. A deep increasingdecreasing-linear neural network for financial time series prediction. Neurocomputing, 347, 5981 .

Armano, G., Marchesi, M., \& Murru, A. 2005. A Hybrid genetic-neural architecture for stock indexes forecasting. Information Sciences, 170(1), 3-33 
518 Ariyo, A. A., Adewumi, A. O., \& Ayo, C. K. 2014. Stock price prediction using the ARIMA

519 model. In 2014 UKSim-AMSS 16th International Conference on Computer Modelling and

520 Simulation, 106-112

521

522 Bark, H. K. K. 1991. Risk, return, and equilibrium in the emerging markets: evidence from the

523 Korean stock market. Journal of Economics and Business, 43(4), 353-362.

524

525 Bustos, O., \& Pomares-Quimbaya, A. 2020. Stock market movement forecast: A Systematic

526 review.Expert Systems with Applications, 156, 113464.

527

528

Cao, J., \& Wang, J. 2019. Stock price forecasting model based on modified convolution neural

529 network and financial time series analysis. International Journal of Communication Systems,

530

531

Damdindorj, T., Rhee, J. H., \& Choi, S. J. 2016. Testing market efficiency with the top-20 index

532 in the Mongolian stock market. Research in Eurasia V13, 1, 131-151.

533

534

Devi, B. U., Sundar, D., \& Alli, P. 2013. An effective time series analysis for stock trend

535 prediction using ARIMA model for nifty midcap-50. International Journal of Data Mining \&

536 Knowledge Management Process, 3(1), 65.

537

538

Ding, X., Zhang, Y., Liu, T., Duan, J. 2015. Deep learning for event-driven stock prediction. In

539 Twenty-fourth international joint conference on artificial intelligence. June

540

541

542

543 Feng, F., Chen, H., He, X., Ding, J., Sun, M., \& Chua, T. S. 2018. Enhancing stock movement

544 prediction with adversarial training. arXiv preprint arXiv:1810.09936.

545

546

Guang, L., Xiaojie, W., \& Ruifan, L. 2019. Multi-scale RCNN model for financial time-series

547 classification. arXiv preprint arXiv:1911.09359.

548

549 Hadavandi, E., Shavandi, H., \& Ghanbari, A. 2010. Integration of genetic fuzzy systems and 550 artificial neural networks for stock price forecasting. Knowledge-Based Systems, 23(8), 800-808.

551

552

553 Han, C., Wang, Y., \& Xu, Y. 2019. Efficiency and multifractality analysis of the Chinese stock

554 market: Evidence from stock indices before and after the 2015 stock market crash. Sustainability, 555 11(6), 1699.

557

Hooshmand, M. K., \& Gad, I. 2020. Feature selection approach using ensemble learning for network anomaly detection.CAAI Transactions on Intelligence Technology, 5(4), 283-293. 
558

559

560

561

562

563

564

565

566

567

568

569

570

571

572

573

574

575

576

577

578

579

580

581

582

583

584

585

586

587

588

589

590

591

592

593

594

595

596

597

Hsu, C. M. 2011. A hybrid procedure for stock price prediction by integrating self-organizing map and genetic programming. Expert Systems with Applications, 8(11), 14026-14036.

Yang, H., Chan, L., \& King, I. 2002. Support vector machine regression for volatile stock market prediction. In International Conference on Intelligent Data Engineering and Automated Learning, 391-396

Jiang, W. 2021. Applications of deep learning in stock market prediction: recent progress. Expert Systems with Applications, 115537. 32(12), e3987.

Joh, S. W. 2003. Corporate governance and firm profitability: evidence from Korea before the economic crisis. Journal of financial Economics, 68(2), 287-322.

Malagrino, L. S., Roman, N. T., \& Monteiro, A. M. 2018. Forecasting stock market index daily direction: A Bayesian Network approach. Expert Systems with Applications, 105, 11-22.

Namasudra, S., Dhamodharavadhani, S., \& Rathipriya, R. 2021. Nonlinear neural network based forecasting model for predicting COVID-19 cases. Neural Processing Letters, 1-21.

Ndichu, S., Kim, S., \& Ozawa, S. 2020. Deobfuscation, unpacking, and decoding of obfuscated malicious JavaScript for machine learning models detection performance improvement. CAAI Transactions on Intelligence Technology, 5(3), 184-192.

O, J., Lee, J. W., Lee, J., \& Zhang, B. T. 2004. Dynamic asset allocation exploiting predictors in reinforcement learning framework. Lecture Notes in Computer Science, 3201, 298-309

O, J., Lee, J., Lee, J. W., Zhang, B. T. 2006. Adaptive stock trading with dynamic asset allocation using reinforcement learning. Information Sciences, 176(15), 2121-2147.

Oncharoen, P., \& Vateekul, P. 2018. Deep learning for stock market prediction using event embedding and technical indicators. In 2018 5th International Conference on Advanced Informatics: Concept Theory and Applications (ICAICTA). 19-24

Refenes, A. N., Zapranis, A., \& Francis, G. 1994. Stock performance modeling using neural networks: a comparative study with regression models. Neural networks, 7(2), 375-388.

Lakshminarayanan, S., Weckman, G. R., Snow, A., \& Marvel, J. 2006. Stock market hybrid forecasting model using neural networks. In IIE Annual Conference Proceedings. Institute of Industrial and Systems Engineers (IISE).

PeerJ Comput. Sci. reviewing PDF | (CS-2021:11:67714:1:1:NEW 27 Jan 2022) 
598

599

600

601

602

603

604

605

606

607

608

609

610

611

612

613

614 Ryoo, H. J., \& Smith, G. 2002. Korean stock prices under price limits: Variance ratio tests of

615

616

617

618

619

620

621

Lee, J. W. 2007. Integrated multiple simulation for optimizing performance of stock trading systems based on neural networks. The KIPS Transactions: PartB, 14(2), 127-134.

Lin, X., Yang, Z., Song, Y. 2011. Intelligent stock trading system based on improved technical analysis and Echo State Network. Expert systems with Applications, 38(9), 11347-11354.

Liu, G., \& Wang, X. 2018. A numerical-based attention method for stock market prediction with dual information. IEEE Access, 7, 7357-7367.

Pang, X., Zhou, Y., Wang, P., Lin, W., \& Chang, V. 2020. An innovative neural network approach for stock market prediction. The Journal of Supercomputing, 76(3), 2098-2118.

Pella, J., \& Masuda, M. 2001. Bayesian methods for analysis of stock mixtures from genetic characters. Fishery Bulletin, 99(1), 151-151.

random walks. Applied Financial Economics, 12(8), 545-553.

Song, Y., \& Lee, J. 2018. Performance evaluation of deep learning stock price by chart type for buying policy verification. In Fuzzy Systems and Data Mining IV, 646-652

Song, Y., Lee, J. W., \& Lee, J. 2020. Development of intelligent stock trading system using pattern independent predictor and turning point matrix. Computational Economics, 1-12. 
Figure 1

MA divergence pattern

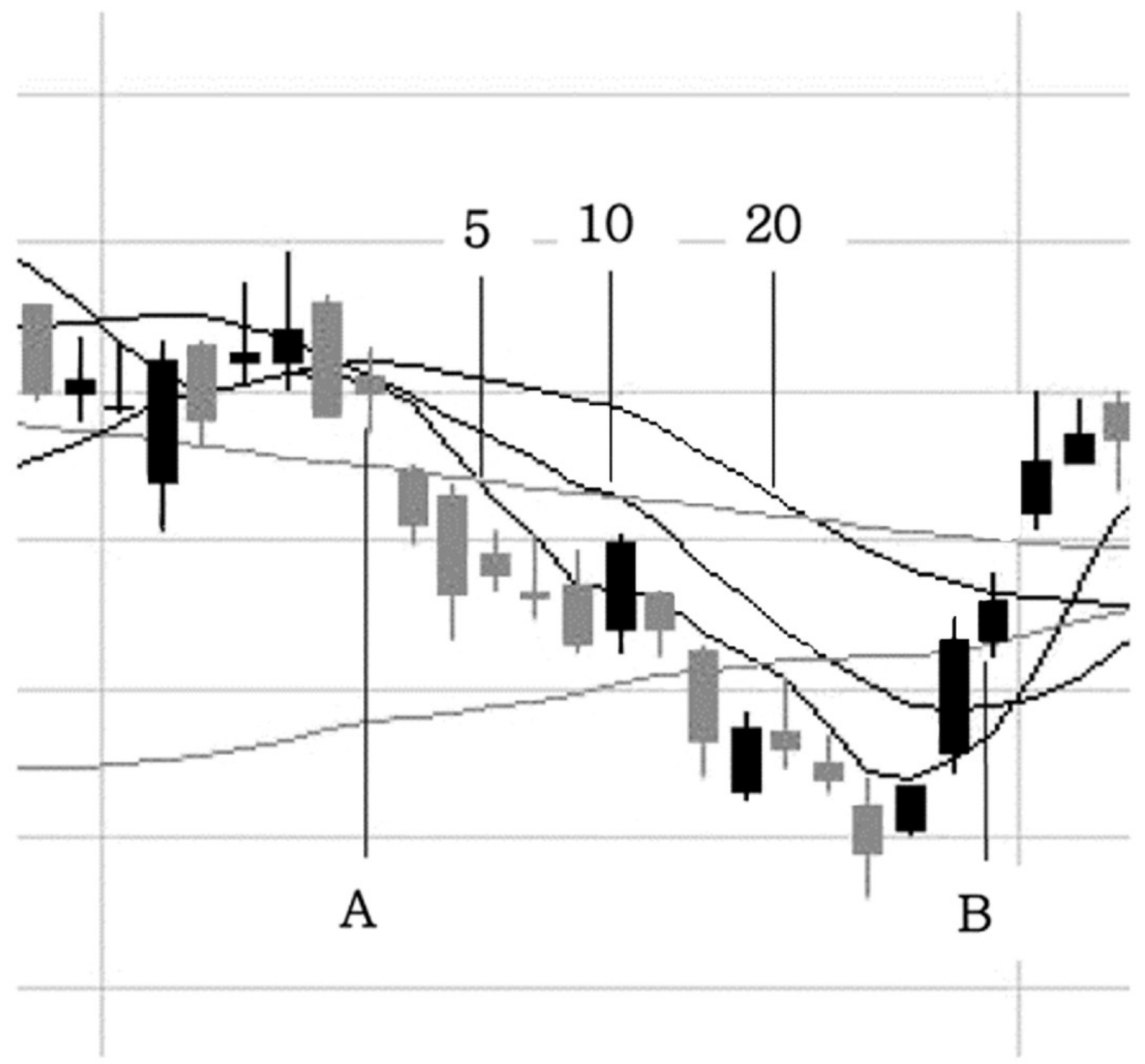


Figure 2

$M A$ reversal to uptrend pattern

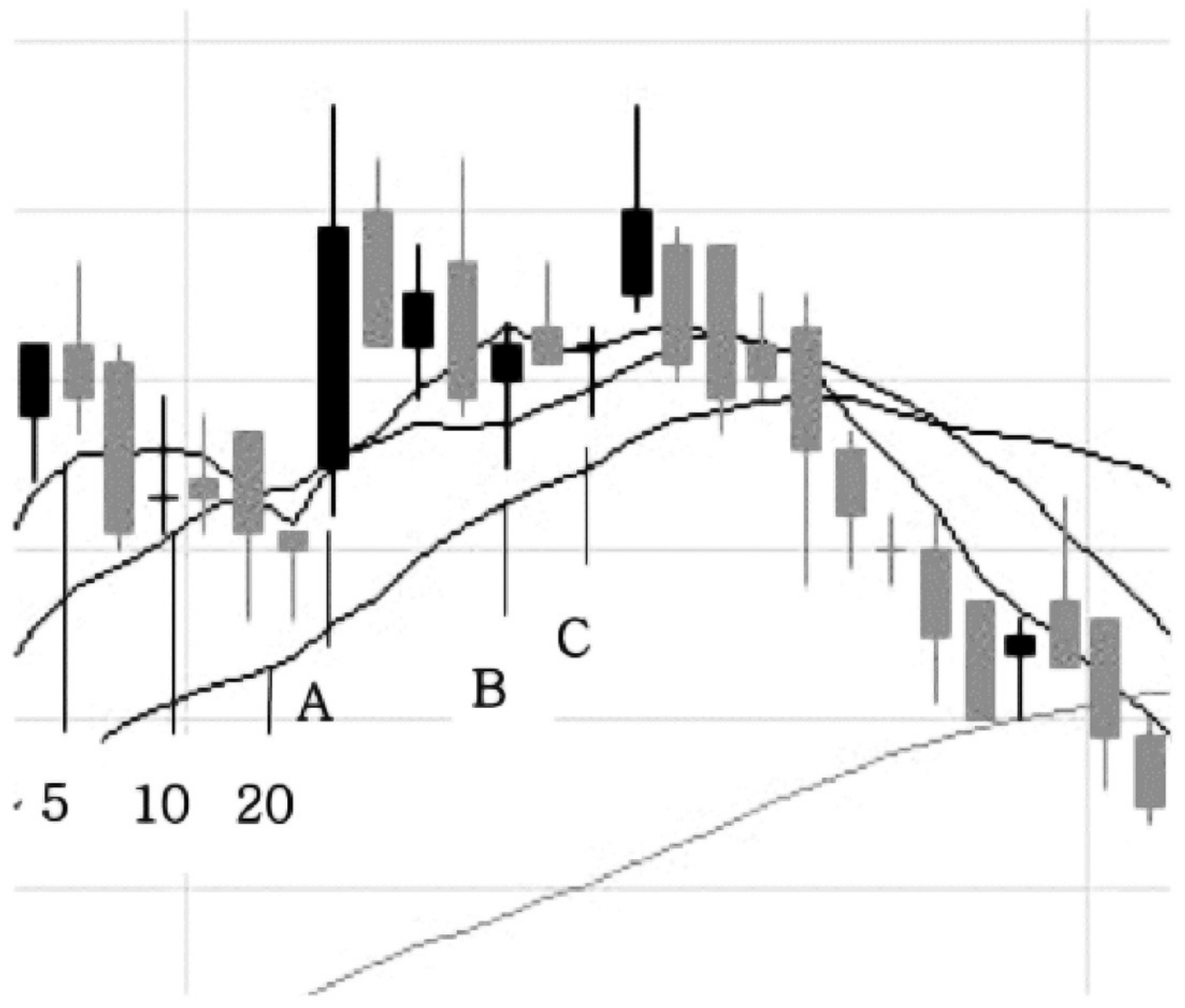


Figure 3

A normal example and counter example of the $\mathrm{p} 1$ pattern

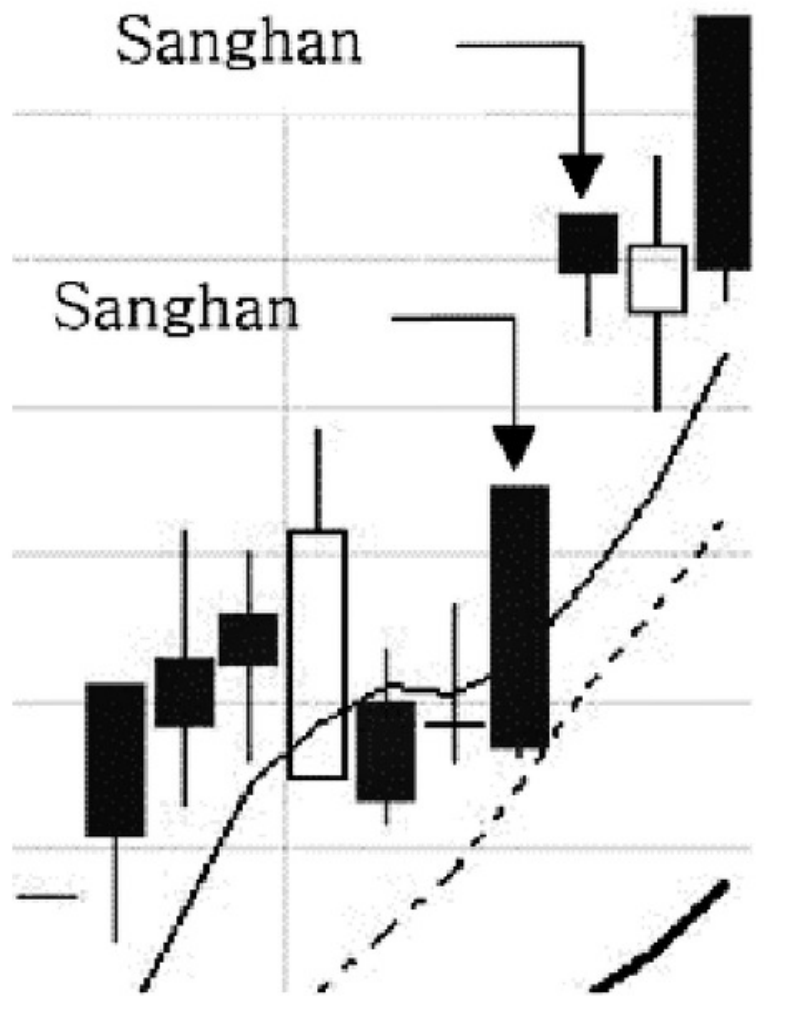

(a)

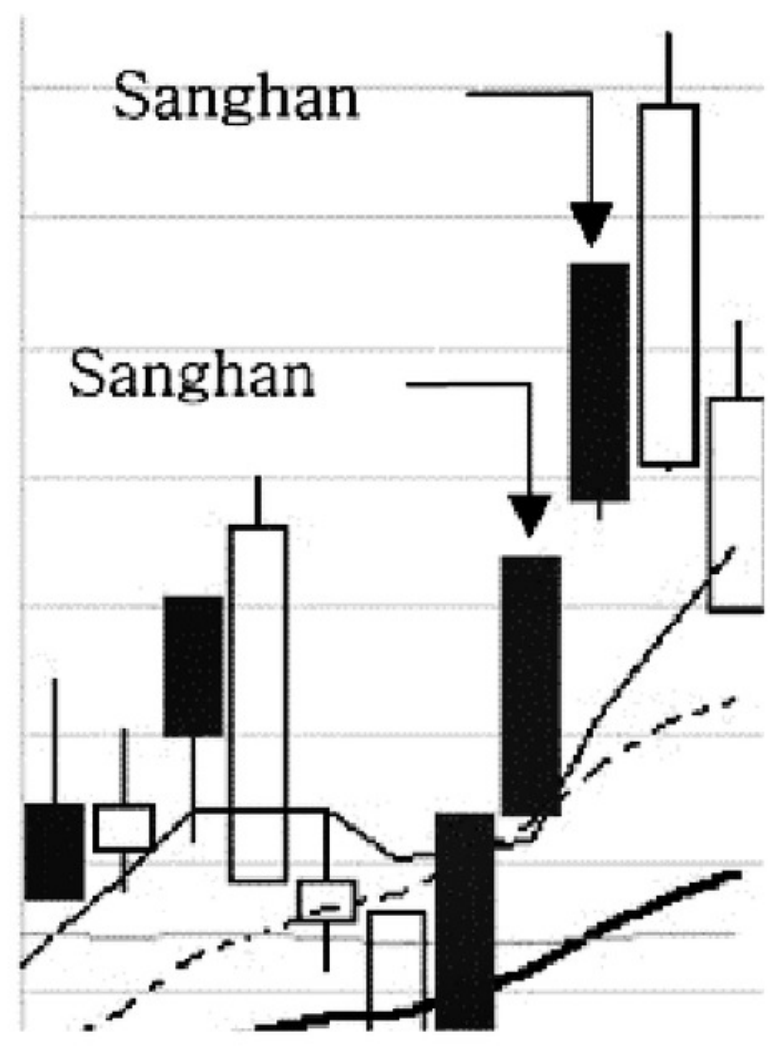

(b) 
Figure 4

A normal example and counter example of the $\mathrm{p} 2$ pattern

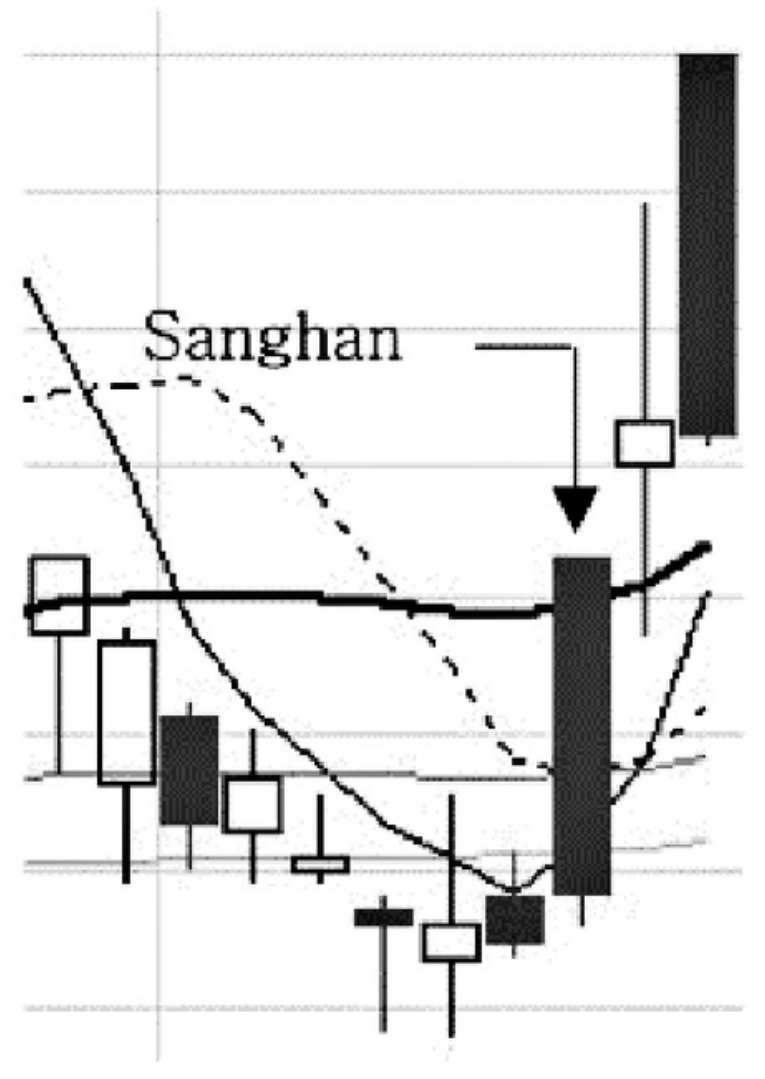

(a)

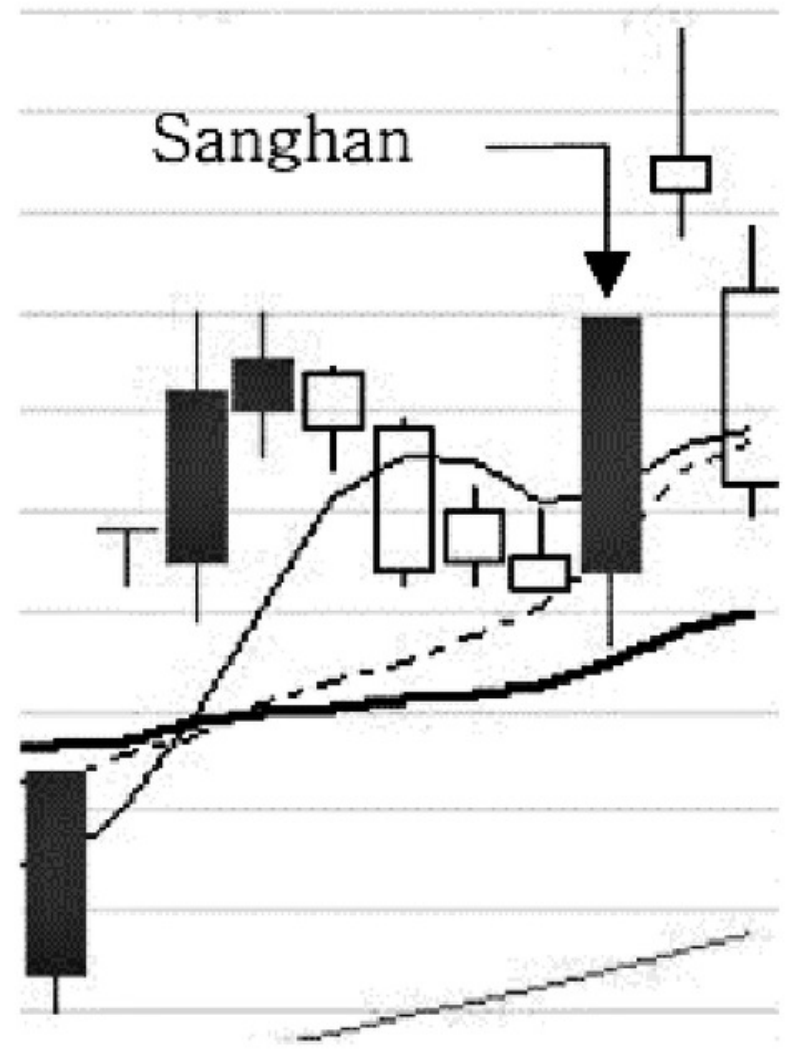

(b) 
Figure 5

A normal example and counter example of the $\mathrm{p3}$ pattern

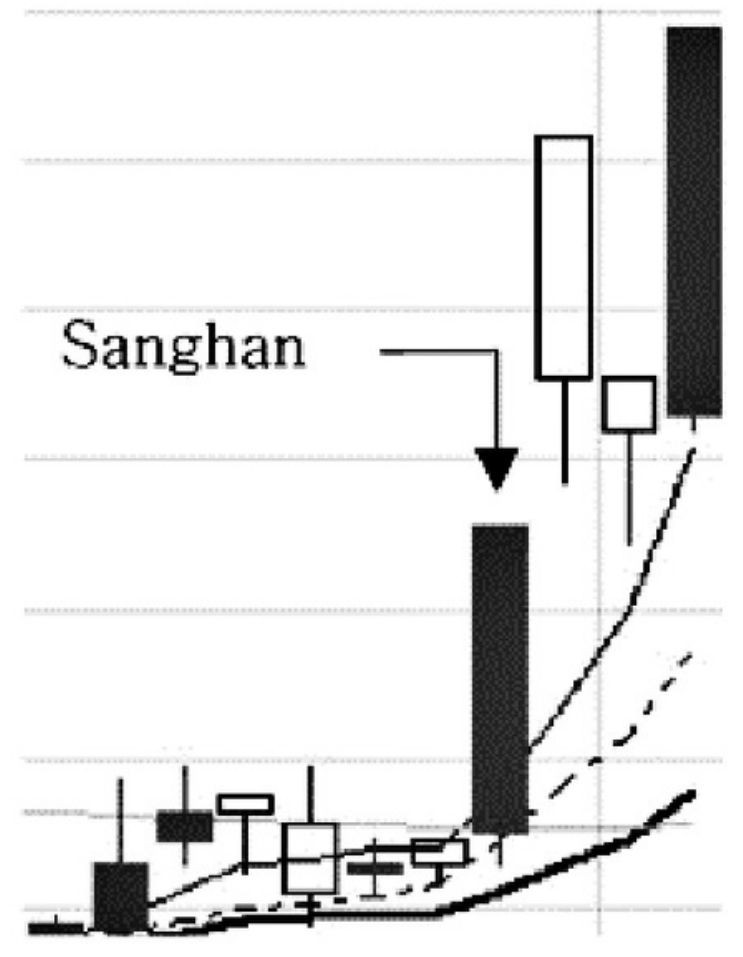

(a)

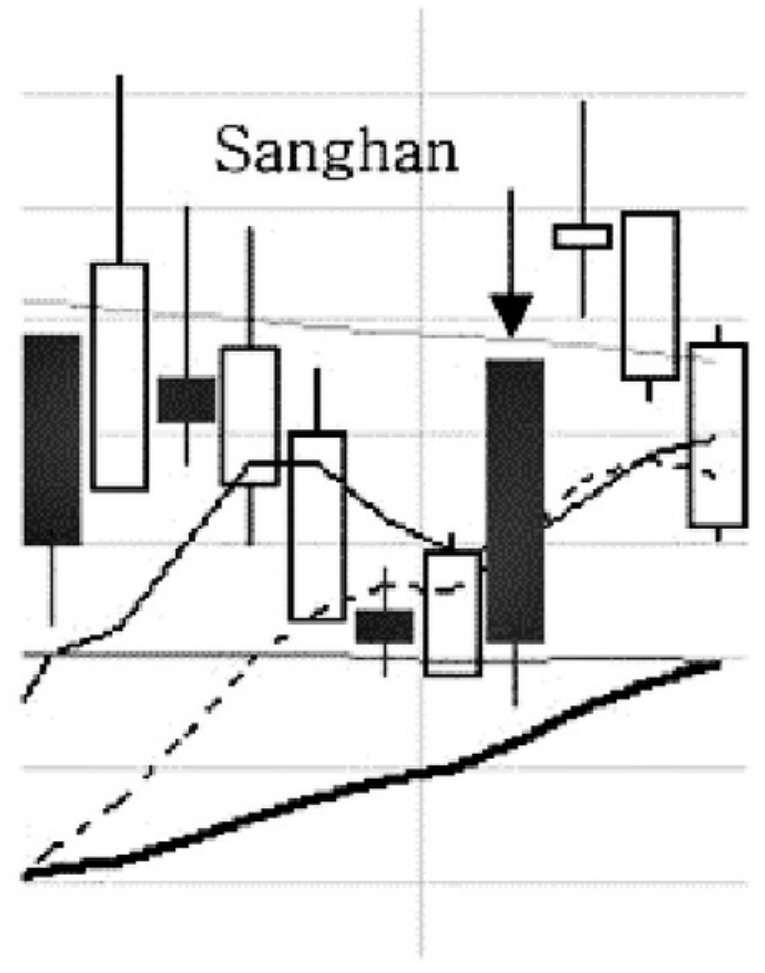

(b) 
Figure 6

Neural network structure and target vector configuration

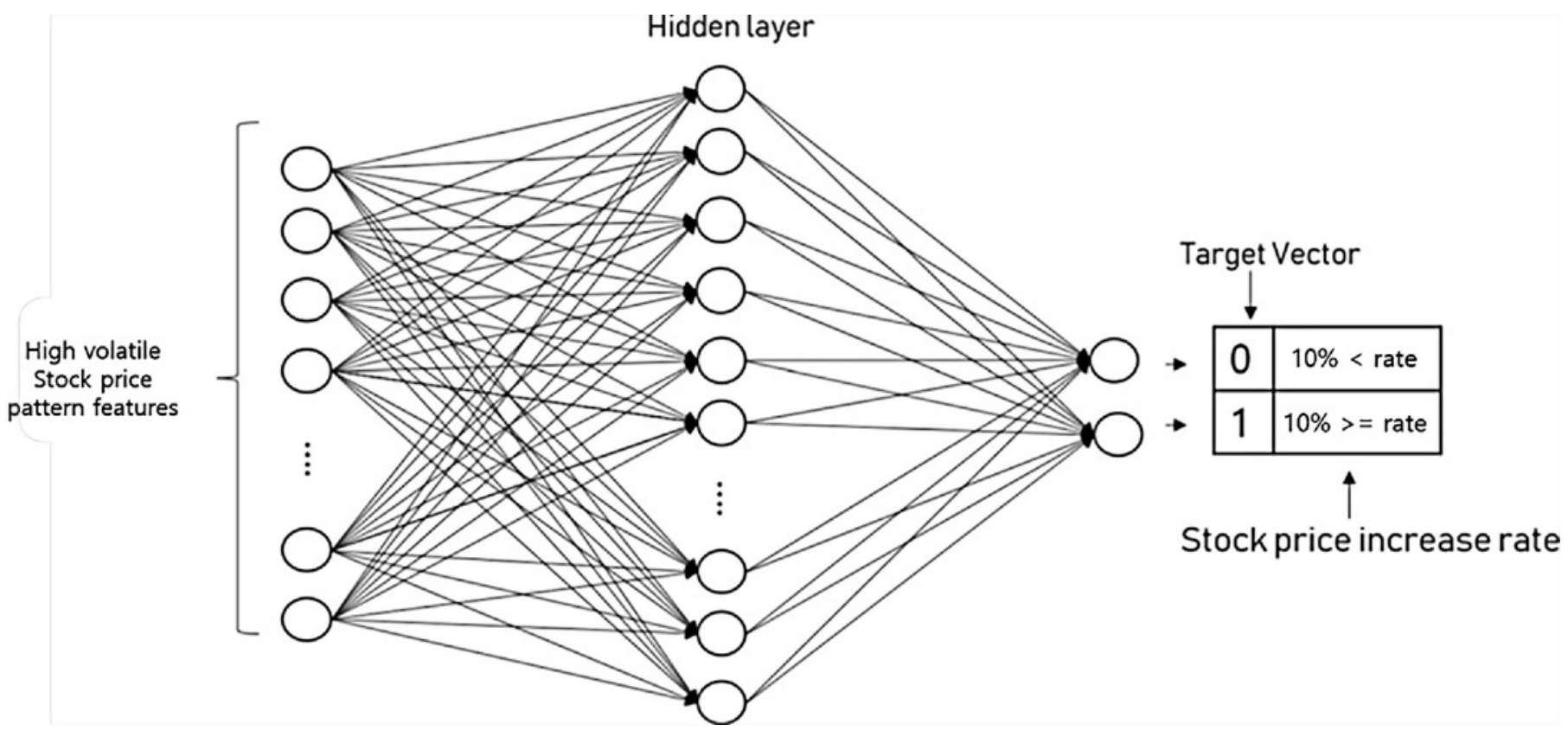


Figure 7

Training and test loss and accuracy graph
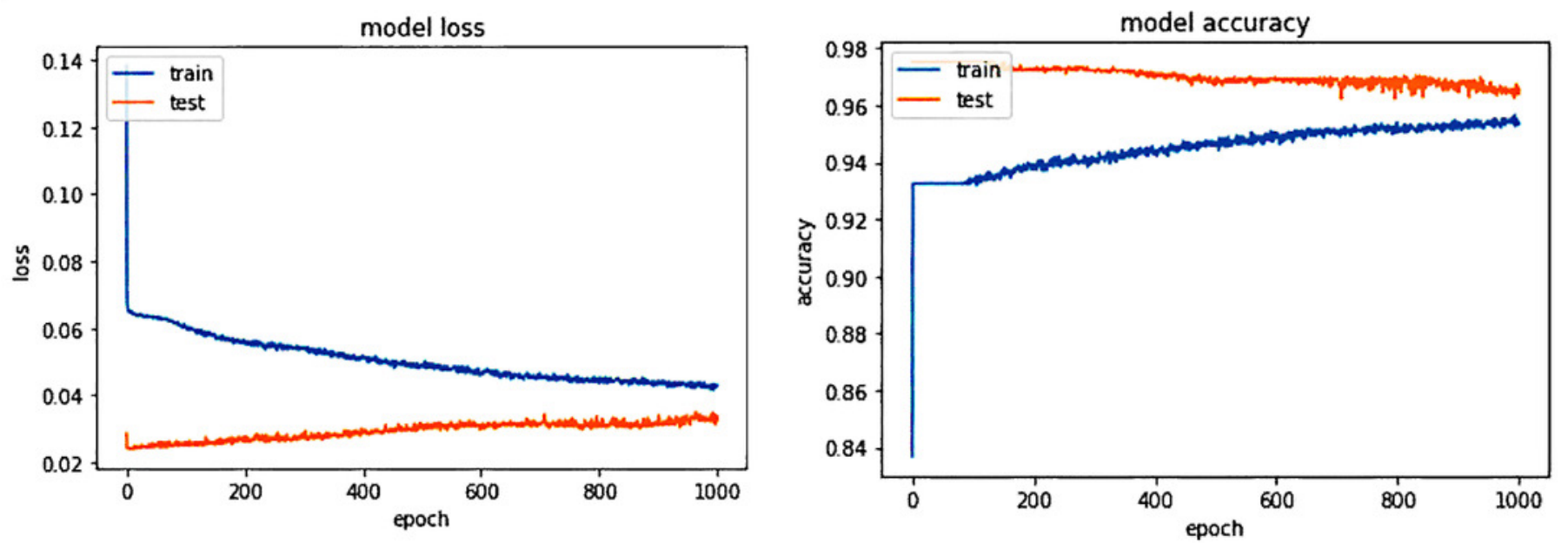
Figure 8

Comparison of the cumulative profit rate between domestic and overseas stock indices and the prediction model

ACCUMULATE PROFIT RATE

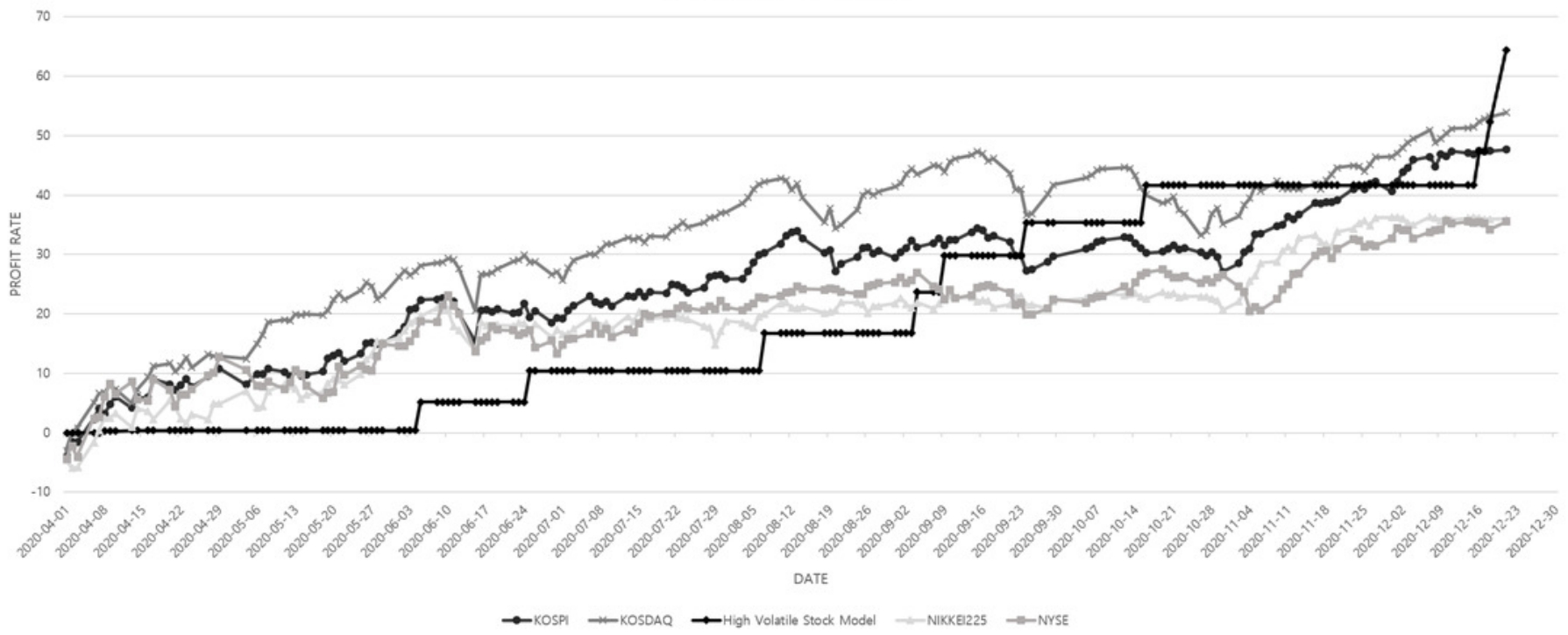




\section{Table 1 (on next page)}

Data set for building the trading system 
1

\begin{tabular}{|c|c|}
\hline Name of dataset & Period \\
\hline Training set & October 2017 to June 2019 \\
\hline Validation set & July 2019 to December 2019 \\
\hline Test Set & January 2020 to April 2020 \\
\hline Fund Simulation Set & May 2020 to December 2020 \\
\hline
\end{tabular}

2 


\section{Table 2 (on next page)}

Model evaluation results 
1

\begin{tabular}{|c|c|c|c|c|}
\hline & Accuracy & Precision & Recall & F1-Score \\
\hline $\begin{array}{c}\text { Model evaluation } \\
\text { Results }\end{array}$ & 0.9623 & 0.9638 & 0.9638 & 0.9638 \\
\hline
\end{tabular}




\section{Table 3 (on next page)}

Comparison of the accuracy between the proposed scheme and other stock filtering schemes 


\begin{tabular}{|c|c|c|c|c|}
\hline & $\begin{array}{c}\text { Resisted } \\
\text { plunge } \\
\text { filtering }\end{array}$ & $\begin{array}{c}\text { Nosedive } \\
\text { filtering }\end{array}$ & $\begin{array}{c}\text { Rise stock } \\
\text { filtering }\end{array}$ & $\begin{array}{c}\text { Our Model } \\
\text { with } \\
\text { highly } \\
\text { volatile stock } \\
\text { price } \\
\text { patterns } \\
\text { filtering }\end{array}$ \\
\hline Accuracy & $72.39 \%$ & $75.11 \%$ & $64.93 \%$ & $96.23 \%$ \\
\hline
\end{tabular}

1 